\title{
Carbohydrate Metabolic Process
}

National Cancer Institute

\section{Source}

National Cancer Institute. Carbohydrate Metabolic Process. NCI Thesaurus. Code

C17933.

Processes concerned with the synthesis, breakdown, and oxidation of carbohydrates in the tissues. 